\title{
Experiment design of the terrestrial laser scanning of elongated objects Marko PEJIĆ ${ }^{*}$, Branko BOŽIĆ ${ }^{1}$, Gligorije PEROVIĆ ${ }^{1}$ \\ ${ }^{1}$ University of Belgrade, Faculty of Civil Engineering, Belgrade, Serbia
}

\begin{abstract}
In high-demanding engineering applications, the latest performance improvements of the terrestrial lasers scanning (TLS) system and price decreasing trend shows the significant potential of this technology. Beside the fact that some scanners have the scanning frequency of over $1.000 .000 \mathrm{~Hz}$, in the engineering applications the accuracy of this survey method plays the key role. Achievement of the satisfactory accuracy of the object modelling using TLS has to be done by experiment designing. This implies the optimization process of the relevant measurements parameters and of the methodology of measurement processing through analysis of the different sources of measurement errors, instrumental precision and performance of the specific TLS, spatial configuration of the object and analysis of the models of registration and georeferencing errors. The proposed methodology of the TLS experiment design is related to the scanning of elongated objects (tunnels, corridors, pipelines, underground passages etc), which generally represent unfavourable cases in providing geodetic measurements of sufficient accuracy and reliability.
\end{abstract}

Keywords: scanning parameters, registration, georeferencing, optimisation 


\section{Introduction}

Realistic and accurate 3D model of objects are beneficial to various fields such as engineering, architecture, urban planning and heritage documentation. The use of TLS measurements in such modelling is gaining an increasing interest due to the very high spatial density of the acquired data and very high geometric accuracy. Basically, TLS employs the laser ranging principle and detection of horizontal and vertical beam deflections during the scanning process. The $3 \mathrm{D}$ position of the scanned point is related to the origin in the scanner electrooptical centre. An additional point attribute is the intensity value of the reflected laser beam.

Today TLS becomes a relevant surveying technique in the field of geodetic engineering. The latest generations of the TLS's have also taken into account the need of the standard surveying procedures, e.g. the feasibility for reliable and accurate levelling, centering, and orienting. It can be stated that TLS is the specific measurement method that shares basic principles of measurements and data processing with tachymetry a terrestrial photogrammetry.

\section{Methodology}

The methodology of the optimization of the scanning parameters starts from the analysis of the different error sources on the scanning accuracy and the defining of the significant influences in the present object configuration conditions. The optimization is performed by taking into account the essential limiting factor such as the incidence angle of the laser beam to the object surface, theoretical contribution of the incidence angle to the signal deterioration and the threshold of the variance components of the measured range.

The quality of scanning is significantly reduced by weak geometry. The incidence angle of the laser beam is the angle between surface normal and laser beam direction. Increasing incidence angles leads to deterioration of the signal to the noise ratio, as seen in [5] and [2]. This is the important limiting factor in the case of scanning the elongated objects.

If we assume that surface affected by laser beam produce uniform reflection of the laser light (Lambertian surface), than the power of the received pulse $P_{r}$ will be:

$$
P_{r}=k P_{t} \cos \alpha_{i}
$$

where $k$ denotes the factor of the range, surface reflectivity and atmospheric properties and $P_{t}$ stands as a emitted pulse power. The incidence angle of the laser beam to the surface normal is $\alpha_{i}$.

If we assume that $k$ and $P_{t}$ have the constant values, then by analyzing the equation (1) it is evident that SNR (signal to noise ratio) factor is consequently decreased by increased $\alpha_{i}$ and wise versa, following the cosines law (Figure 1).

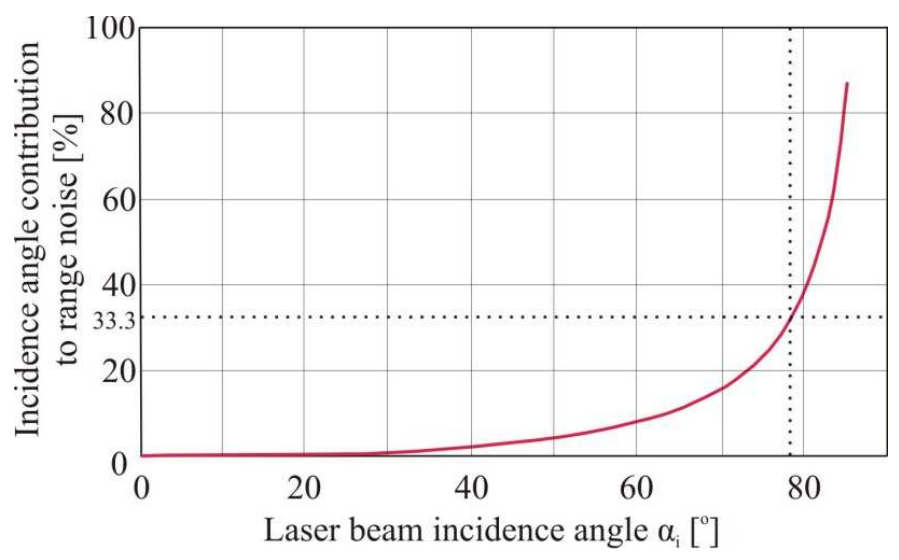

Figure 1. Signal noise induced by the incidence angle

Incidence angle contribution component $B^{2}$ of the overall range variance can be considered as insignificant at the selected level of confidence $\alpha$, if ([7] and [6]):

$$
B^{2}<\frac{2 \alpha-\alpha^{2}}{(1-\alpha)^{2}} \cdot A^{2}
$$

where $A^{2}$ stands as the variance component containing other error sources of the measured range.

For $\alpha=0.05$ confidence interval, the standard deviation component $B$ is not significant if:

$$
B<\frac{1}{3} A
$$

The contribution of the theoretical range noise caused by the incidence angle is shown on Figure 1 . The maximal allowed incidence angle is $78^{\circ}$. That angle will contribute to the overall noise by $33 \%$, which can be considered as insignificant in $95 \%$ of cases (3).

If we assume that the inner shield of the tunnel is free from the shadowing effects on the scanning data, then the optimal size of the TLS shifting along the tunnel alignment can be calculated by the incidence angle criteria. 
When registering a sequence of scans along the tunnel, the errors of global registration may be rather large. This happens due to the error propagation from the first scan to the subsequent scans ([4], [1]). The proposed solution for this problem is the arbitrary georeferencing in two steps. The scans need to be grouped into sections. The criterion for the number of registered scans in one section is the size of the transformation residuals and the given accuracy of the tunnel 3D model. This approach holds the final point cloud geometric distortion, due to scans registration errors multiplication, to the given limits.

Each proposed georeferencing method needs a control survey network. This network needs to provide a reliable and accurate base for further georeferencing of the scanned data.

\section{Case study}

TLS experiment design that includes the optimization of the scanning parameters is conducted based on the case geometry of the 12 railway single-track tunnels located at the NikšićPodgorica railway (Figure 2). On this test example, the location of the TLS stations, allowed scanning distance and angular scanning resolution are designed based on the requested 3D tunnel model accuracy: $\sigma_{\mathrm{m}}=2 \mathrm{~cm}$.

The tunnels are $6.2 \mathrm{~m}$ high and $5.5 \mathrm{~m}$ wide in average. The maximal allowed scanning distance is $13 \mathrm{~m}$, and consequently the optimal size of the scanner shifting along the tunnel alignment is $26 \mathrm{~m}$. Optimal scanning resolution is 60" using approach from [3]. At a $13 \mathrm{~m}$ range from the scanner the minimal desirable point density is 1 point per square centimetre.

Given the scanner positions at $26 \mathrm{~m}$ intervals, it is designed that on every third TLS station, one control point will be total station centering point (St1, St2...Stn). On the same alignment section two scanner target control points are marked on the tunnel wall (M1, M2...Mn). Additional control points that improve the reliability factors of the measurements and check the quality of the georeferencing are marked on the rail bolts (K1, $\mathrm{K} 2 \ldots \mathrm{Kn}$ ). The positions of these points are at half the alignment distance from the adjacent St and $\mathrm{M}$ control points (Figure 3.).

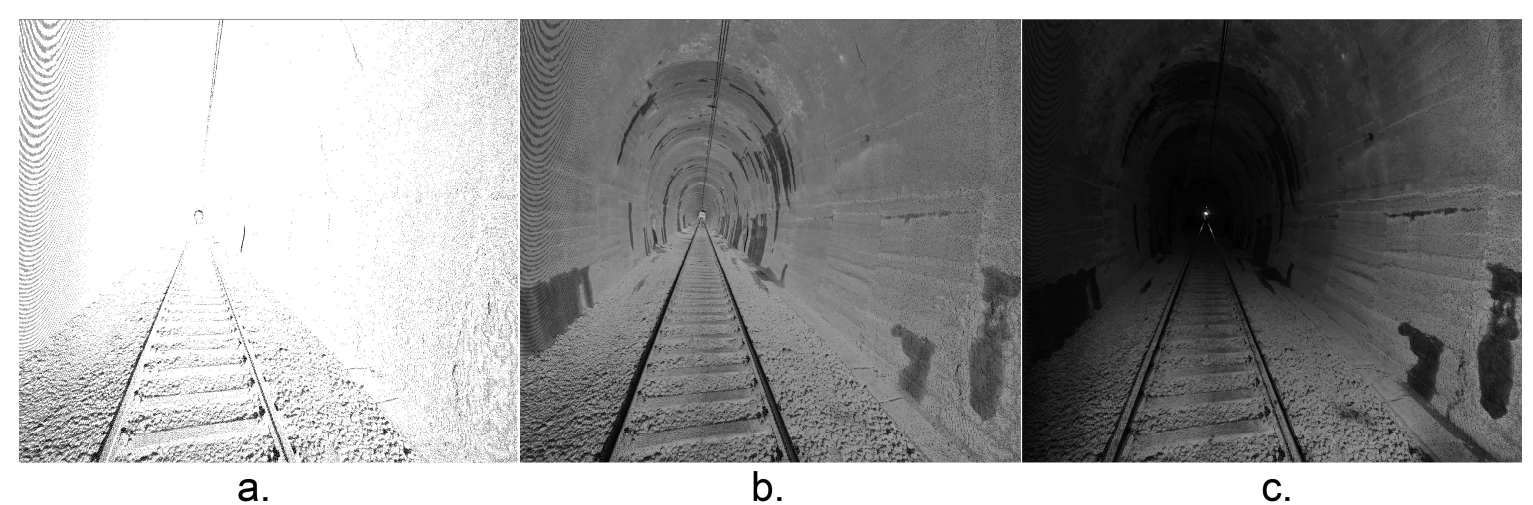

Figure 2. Point cloud of tunnel. With no point attribute (a.), with intensity (b.) and with RGB point attribute (c.).

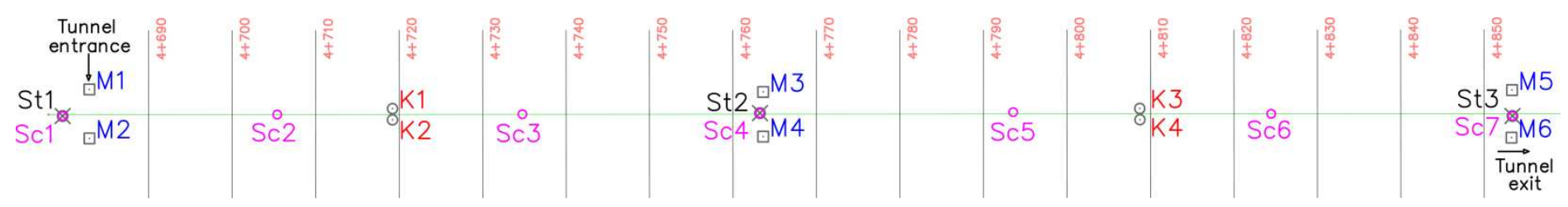

Figure 3. Control network of the elongated objects laser scanning

For the $1260 \mathrm{~m}$ long tunnel example, efficiency of the arbitrary georeferencing approach is shown as opposed to the classical indirect approach. This creates the possibility of achieving the high geometric accuracy of the engineering object model, even in the environment unfavourable for the geodetic measurements. Using criteria presented in the methodology section, standard error of the georeferencing, using arbitrary georeferencing method, in the longest example tunnel is $10.6 \mathrm{~mm}$ 
(Table 1), while residuals of the standard indirect georeferencing approach goes up to $1 \mathrm{~m}$.

Table 1: Residuals of the arbitrary georeferencing $(\Delta) .90 \%$ of the sample errors fall into a theoretical $1 \sigma(2$ $\mathrm{cm})$ confidence interval. Data for 1.26 km long tunnel "Budoš".

\begin{tabular}{|c|c|c|c|c|c|c|c|}
\hline Point & $\begin{array}{c}\Delta_{Y} \\
{[\mathrm{~mm}]}\end{array}$ & $\begin{array}{c}\Delta_{X} \\
{[\mathrm{~mm}]}\end{array}$ & $\begin{array}{c}\Delta_{H} \\
{[\mathrm{~mm}]}\end{array}$ & $\begin{array}{c}\Delta_{3 D} \\
{[\mathrm{~mm}]}\end{array}$ & Segment & Scene & $\begin{array}{c}\text { Segment } \Delta_{3 D} \\
{[\mathrm{~mm}]}\end{array}$ \\
\hline$M 1$ & 0.3 & 0.5 & -0.7 & 0.9 & \multirow{4}{*}{1} & \multirow{4}{*}{ 02-06 } & \multirow{4}{*}{4.8} \\
\hline$M 2$ & -0.1 & -1.5 & 0.7 & 1.7 & & & \\
\hline M3 & 6.5 & 1.1 & 0.6 & 6.6 & & & \\
\hline M4 & -6.7 & 0.0 & -0.6 & 6.7 & & & \\
\hline M3 & 5.1 & -0.6 & -12.3 & 13.3 & \multirow{8}{*}{2} & \multirow{8}{*}{$06-15$} & \multirow{8}{*}{16.1} \\
\hline M4 & -8.2 & -1.6 & -17.9 & 19.7 & & & \\
\hline M5 & 10.0 & -0.1 & 20.9 & 23.2 & & & \\
\hline M6 & -3.9 & 1.6 & 11.9 & 12.6 & & & \\
\hline$M 7$ & 3.7 & -0.6 & 8.0 & 8.8 & & & \\
\hline$M 8$ & -5.4 & -1.9 & 15.9 & 16.9 & & & \\
\hline M9 & 4.8 & 2.9 & -16.8 & 17.7 & & & \\
\hline M10 & -6.0 & 0.3 & -9.7 & 11.4 & & & \\
\hline M9 & 5.6 & -3.1 & 2.2 & 6.8 & \multirow{4}{*}{3} & \multirow{4}{*}{$15-18$} & \multirow{4}{*}{6.3} \\
\hline M10 & -4.7 & -1.5 & -2.2 & 5.4 & & & \\
\hline M11 & 4.9 & 4.0 & -2.4 & 6.7 & & & \\
\hline M12 & -5.8 & 0.6 & 2.4 & 6.3 & & & \\
\hline M11 & 5.3 & 0.2 & -2.0 & 5.7 & \multirow{4}{*}{4} & \multirow{4}{*}{$18-21$} & \multirow{4}{*}{6.5} \\
\hline$M 12$ & -5.3 & -0.2 & 2.0 & 5.7 & & & \\
\hline$M 13$ & 7.1 & -0.5 & 1.4 & 7.2 & & & \\
\hline M14 & -7.1 & 0.5 & -1.4 & 7.3 & & & \\
\hline M13 & 6.8 & -1.2 & -0.2 & 6.9 & \multirow{4}{*}{5} & \multirow{4}{*}{$21-25$} & \multirow{4}{*}{7.7} \\
\hline M14 & -7.1 & 1.8 & 0.2 & 7.3 & & & \\
\hline M15 & 8.3 & -0.1 & 0.2 & 8.3 & & & \\
\hline M16 & -8.1 & -0.6 & -0.2 & 8.1 & & & \\
\hline M15 & 10.8 & -5.9 & -2.8 & 12.6 & \multirow{6}{*}{6} & \multirow{6}{*}{$25-31$} & \multirow{6}{*}{10.1} \\
\hline M16 & -6.0 & -4.3 & 1.4 & 7.5 & & & \\
\hline M17 & 7.3 & -3.2 & 2.7 & 8.4 & & & \\
\hline M18 & -13.7 & 4.4 & 0.5 & 14.4 & & & \\
\hline M19 & 6.9 & 5.3 & 0.2 & 8.7 & & & \\
\hline M20 & -5.2 & 3.7 & -2.0 & 6.7 & & & \\
\hline M19 & 9.1 & -5.5 & -5.0 & 11.7 & \multirow{6}{*}{7} & \multirow{6}{*}{$31-37$} & \multirow{6}{*}{9.1} \\
\hline$M 20$ & -3.1 & -6.0 & -2.0 & 7.1 & & & \\
\hline$M 21$ & -0.1 & 0.1 & 7.3 & 7.3 & & & \\
\hline$M 22$ & -7.2 & -2.6 & 7.4 & 10.6 & & & \\
\hline$M 23$ & -1.7 & 5.5 & -2.4 & 6.3 & & & \\
\hline$M 24$ & 3.0 & 8.5 & -5.2 & 10.4 & & & \\
\hline$M 23$ & -2.3 & -8.5 & 7.1 & 11.3 & \multirow{8}{*}{8} & \multirow{8}{*}{$37-46$} & \\
\hline$M 24$ & 2.6 & -5.6 & 10.7 & 12.4 & & & \\
\hline$M 25$ & 2.5 & -4.4 & -14.3 & 15.1 & & & \\
\hline M26 & 3.6 & -1.2 & -11.9 & 12.4 & & & \\
\hline$M 27$ & -3.3 & -4.7 & 0.0 & 5.8 & & & 11.6 \\
\hline$M 28$ & 1.4 & -0.5 & 0.7 & 1.6 & & & \\
\hline M29 & 0.2 & 14.6 & 3.6 & 15.0 & & & \\
\hline M30 & -4.7 & 10.3 & 4.0 & 12.0 & & & \\
\hline
\end{tabular}

Tunnel modelling and profiling can be conducted once the optimal quality of the geometry of the point cloud data is satisfied. The triangular mesh of the scanned tunnels is analyzed with 


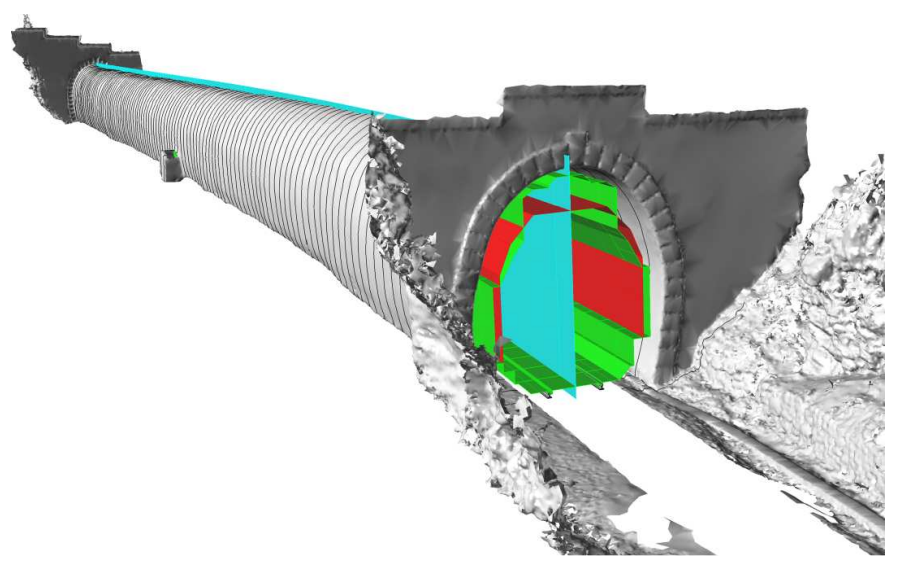

Figure 4. Tunnel and train 3D model

\section{Conclusion}

The design and optimization of geodetic measurements traditionally have been used in engineering. The emergence of new technologies, such as laser scanning, involves a somewhat modified approach to this issue. Despite the specifics of this method of collecting spatial data, especially in their processing and interpretation, the basic engineering approach remains the same and relies on statistical analysis and the theory of errors in geodetic measurements.

\section{Acknowledgement}

This work is part of the technological development project No TR36009: "The Application of GNSS and LIDAR Technology for Infrastructure Facilities and Terrain Stability Monitoring" funded by the Ministry of Education, Science and Technological Development of the Republic of Serbia.

\section{References}

[1]Bornaz, L., Lingua, A., Rinaudo, F., 2003. Multiple scan registration in LIDAR close-range applications. Proceedings of the ISPRS International Workshop WG V/4 and INTCOM III/V "Vision Techniques for
Digital Architectural and Archaeological Archives". Ancona, Italy, July $1-3$.

[2]Lichti, D.D., 2007. Error modelling, calibration and analysis of an AM CW terrestrial laser scanner system. ISPRS Journal of Photogrammetry and Remote Sensing 61 (5), 307-324

[3] Lindenbergh, R., Pfeifer, N., Rabbani, R., 2005. Accuracy analysis of the Leica HDS3000 and feasibility of tunnel deformation monitoring. IAPRS Vol XXXVI, 3/W3, Proceedings of Laserscanning 2005, Enschede, The Netherlands.

[4] Reshetyuk, Y., 2009. Self-calibration and direct georeferencing in terrestrial laser scanning. Doctoral thesis in Infrastructure, Geodesy. Royal Institute of Technology, Division of Geodesy. Stockholm.

[5] Soudarissanane, S., Lindenbergh, R., Menenti, M., Teunissen, P., 2011. Scanning geometry: Influencing factor on the quality of terrestrial laser scanning points. ISPRS Journal of Photogrammetry and Remote Sensing. Volume 66, Issue 4, pp 389-399.

[6]Perović G., 1985. Neglectivity of systematic errors. Sixth International Meeting of A.E.A, Health Econometrics No 2. Rotterdam, 16-17 December 1985, 158-161.

[7]Činklović, N., 1978. Analysis and a-priori accuracy estimation of precise geodetic measurements, Monograph. Faculty of Civil Engineering, Belgrade (in Serbian). 